Psychological Medicine

cambridge.org/psm

\section{Invited Letter Rejoinder}

Cite this article: Chien Y-L (2021). Response to the commentary by Bankart and Brugha. Psychological Medicine 1-2. https://doi.org/ 10.1017/S0033291721004578

Received: 24 September 2021 Revised: 8 October 2021

Accepted: 21 October 2021

Author for correspondence:

Yi-Ling Chien, E-mail: ylchien@hotmail.com

\title{
Response to the commentary by Bankart and Brugha
}

Yi-Ling Chien (1)

Department of Psychiatry, National Taiwan University Hospital and College of Medicine, Taipei, Taiwan

Dear Editor,

In response to the commentary by Bankart and Brugha (2021), we appreciate the opportunity to address the concerns raised by the authors.

To clarify, the research summarized in Chien et al. (2021) was a single-site study, utilizing an evidence-based, manualized intervention protocol (Laugeson, 2017), which was translated into Mandarin Chinese and culturally modified with the developer's permission, and ultimately published in Mandarin Chinese (ISBN 978-986-357-130-8). Treatment was delivered to fidelity by the intervention team following the published intervention protocol.

The trial is registered on ClinicalTrial.gov (NCT05054829). The study protocol was approved by the Research Ethics Committee of National Taiwan University Hospital (no. 201612185RINC). The primary outcome of the study was social functioning assessed by the Social Responsiveness Scale (SRS). The significance level was set at $p<0.05$. The outcome was evaluated by as-treated analysis.

As for "minimum clinically important" effect size, there is a lack of research standards to date, particularly for psychotherapeutic approaches (Cuijpers, Turner, Koole, van Dijke \& Smit, 2014). Lemieux et al. (2007) demonstrated that multiplying the pooled baseline standard deviation (s.D.) by 0.2 as the minimal clinically important difference corresponds to the smallest effect size. Cuijpers et al. (2014) suggested a tentative clinical relevance cutoff of standardized mean difference (effect size) 0.24 for psychotherapeutic intervention. As for social skill training in autism spectrum disorder (ASD), previous studies suggested that social skill training improves social deficits in ASD children, with effect sizes in the medium range $(d=0.47$ 0.51 ). A recent meta-analysis of in-person and technological interventions showed that the effect sizes of face-to-face social skills training are in the medium to high range $(g=0.81)$ (Soares et al., 2021). Yet, effect sizes may vary with assessment tools and clinical populations, such as age and intellect. Unlike the abundant treatment trials for ASD children, adult intervention research is still limited. Thus, more research is certainly needed to define the clinical relevance cutoffs for social skills training outcomes in ASD adults. Adopting two clinical importance criteria proposed by Cuijpers et al. (2014) (effect size $d>0.24$ ) and Lemieux et al. (2007) (mean differences > pooled baseline S.D. multiplied by 0.2; i.e., SRS total scores: self-reported $28.7 \times 0.2=5.74$, coach-reported $22.1 \times 0.2=4.42$ ), social functioning measured on the SRS total and social communication subscale in this study (effect size: self-reported $d=0.41>0.24$, coach-reported $d=0.73>0.24$; mean difference: self-reported $11.7>5.74$, coach-reported $15.8>4.42$ ) were considered as clinical important differences following the social skill training.

With regard to the randomization procedures, simple randomization (rather than block randomization) was applied within each stratum (i.e. male or female). For allocation of the participants, we used a computer-generated list of random numbers. Participants of each sex were randomly assigned to the PEERS intervention group or treatment-as-usual control group following simple randomization procedures. As for implementation, the research team enrolled participants based on eligibility criteria (Chien et al., 2021), while a trained research assistant generated the allocation sequence and randomized participants using the above procedures.

As for the adjustment made to the familywise error rate, for the main findings of mixed model summarized in original Table 2 (Chien et al., 2021), if Bonferroni correction was adopted (significance threshold $0.05 / 15=0.0033$ ), self-reported SRS social emotion, SRS total scores, Empathy Quotient total scores, social skills knowledge, and communication and social reciprocity of the autism diagnostic observation schedule passed the corrected threshold. If adjusted by false discovery rate (FDR), most domains remained significant (FDR $q<0.05$ ) except self-reported AQ total scores, SRS social awareness and coach-reported SRS total scores.

For sample size and power calculation, in the mixed model with repeated measures, a total sample of 82 had statistical power at 0.8 to detect group differences with an effect size $d>0.27$ $(\alpha<0.05)$. For paired $t$ test, 41 pairs of follow-up data had statistical power at 0.8 to detect group differences with effect size $d>0.45$. Thus, this sample size may be adequate to detect medium to large effects, but is underpowered to detect small effect differences (e.g. $d<0.27$ 
in mixed model or $d<0.45$ in paired $t$ test). Thus, we agree that the findings may be presented as preliminary and provide the grounds for a larger, definitive study.

We appreciate the comments, and would like to echo that autism researchers work in collaboration with people with autism to engage in a joint effort to seek greater consensus on intervention outcome prioritization.

Conflict of interest. The author declares no competing interest.

\section{References}

Bankart, J., \& Brugha, T. (2021). Social skills interventions in young adults with autism spectrum disorder: Comment on Chien et al. (2021). Psychological Medicine, 12, 1-2. doi: 10.1017/S0033291721003998, Online ahead of print.

Chien, Y. L., Tsai, W. C., Chen, W. H., Yang, C. L., Gau, S. S., Soong, W. T., ... Chiu, Y. N. (2021). Effectiveness, durability, and clinical correlates of the
PEERS social skills intervention in young adults with autism spectrum disorder: The first evidence outside North America. Psychological Medicine, 1-11. doi: $10.1017 /$ S0033291721002385, Online ahead of print.

Cuijpers, P., Turner, E., Koole, S., van Dijke, A., \& Smit, F. (2014). What is the threshold for a clinically relevant effect? The case of major depressive disorders. Depression and Anxiety, 31(5), 374-378. doi: 10.1002/da.22249.

Laugeson, E. (2017). PEERS ${ }^{\circledR}$ for young adults: Social skills training for adults with autism spectrum disorder and other social challenges. New York: Routledge.

Lemieux, J., Beaton, D. E., Hogg-Johnson, S., Bordeleau, L. J., \& Goodwin, P. J. (2007). Three methods for minimally important difference: No relationship was found with the net proportion of patients improving. Journal of Clinical Epidemiology, 60(5), 448-455. doi: 10.1016/j.jclinepi.2006.08.006

Soares, E. E., Bausback, K., Beard, C. L., Higinbotham, M., Bunge, E. L., \& Gengoux, G. W. (2021). Social skills training for autism spectrum disorder: A meta-analysis of in-person and technological interventions. Journal of Technology in Behavioral Science, 6, 166-180. doi: 10.1007/ s41347-020-00177-0 